\title{
Cation non-stoichiometry in yttrium-doped barium zirconate: phase behavior, microstructure, and proton conductivity
}

\author{
Yoshihiro Yamazaki, Raul Hernandez-Sanchez and Sossina M. Haile*
}

\author{
Received 25th June 2010, Accepted 9th July 2010 \\ DOI: 10.1039/c0jm02013c
}

Recent literature indicates that cation non-stoichiometry in proton-conducting perovskite oxides $\left(\mathrm{ABO}_{3}\right)$ can strongly influence their transport properties. Here we have investigated A-site nonstoichiometry in $\mathrm{Ba}_{1-x} \mathrm{Zr}_{0.8} \mathrm{Y}_{0.2} \mathrm{O}_{3-\delta}$, a candidate electrolyte material for fuel cell and other electrochemical applications. Synthesis is performed using a chemical solution approach in which the barium deficiency is precisely controlled. The perovskite phase is tolerant to barium deficiency up to $x=0.06$ as revealed by $\mathrm{X}$-ray diffraction analysis, but accommodates the non-stoichiometry by incorporation of yttrium on the A-site. The dopant partitioning can explain the decrease in cell constant with increasing $x$, the decrease in proton conductivity (the latter as measured by a.c. impedance spectroscopy under humidified atmosphere), and the decrease in grain size in the sintered compacts. Within the single-phase region barium deficiency also has a detrimental impact on grain boundary conductivity, as a result both of the decreased grain size, leading to a higher number density of grain boundaries and of an increased per boundary resistivity. At higher values of $x$, a two phase system is observed, with yttria appearing as the predominant secondary phase and the barium zirconate reverting to an undoped composition. From the relative concentrations of the observed phases and their lattice constants, the ternary phase behavior at $1600^{\circ} \mathrm{C}$ (the sintering temperature) is generated. Both the bulk and grain boundary conductivities are sharply lower in the two-phase system than in the single phase compositions. The control over processing conditions demonstrates that it is possible to reproducibly prepare large-grained, stoichiometric $\mathrm{BaZr}_{0.8} \mathrm{Y}_{0.2} \mathrm{O}_{3-\delta}$ with a total conductivity of $1 \times 10^{-2} \mathrm{Scm}^{-1}$ at $450{ }^{\circ} \mathrm{C}$, while revealing the mechanisms by which barium deficiency degrades properties.

\section{Introduction}

Many oxides, in particular those with the perovskite crystal structure, have been observed to transport charge through the motion of protons. ${ }^{1-4}$ In these systems, proton incorporation is generally understood to occur by a two step process. Trivalent element doping on the tetravalent site of the $\mathrm{A}^{2+} \mathrm{B}^{4+} \mathrm{O}_{3}$ perovskite creates, as a consequence of electroneutrality requirements, oxygen vacancies in the system. Upon exposure to water vapor, hydroxyl groups are incorporated at the oxygen vacancies and an equal number of additional protons become associated with occupied oxygen sites. Once incorporated, protons can migrate between oxygen ions, leading to the observed protonic conductivity.

Barium zirconate, specifically yttrium-doped $\mathrm{BaZrO}_{3}$, has attracted particular attention amongst proton conducting oxides because of its chemical stability, mechanical robustness and, when appropriately processed, high bulk proton conductivity. ${ }^{5-8}$ Challenges surrounding the use of this material center on (i) the difficulty of attaining reproducible properties, (ii) the inherently high grain boundary resistance, and (iii) the high number-density of grain boundaries due to poor grain growth characteristics. These challenges are furthermore interrelated because processing approaches that lead to large-grained materials, such as prolonged sintering at extreme temperatures, often result in

Materials Science, California Institute of Technology, 1200 E California Blvd, Pasadena, CA, 91125, USA.E-mail: smhaile@caltech.edu lowered bulk or grain interior conductivities. Alternative approaches such as reactive sintering, ${ }^{8}$ introduction of sintering aids such as $\mathrm{ZnO}, \mathrm{CoO}$ or $\mathrm{NiO},{ }^{9,10}$ and the combination thereof ${ }^{11}$ show promise as reproducible routes to materials with both high bulk conductivity and large grains, leading to a total high conductivity. However, the stability of transition metal oxide additives under reducing conditions and their impact on the ionic transference number have yet to be seriously explored. These concerns suggest that reactive sintering in the absence of additives ${ }^{8}$ may ultimately find the greatest utility.

The question that is addressed in the present work is the origin of the wide scatter in reported proton conductivities in yttrium doped barium zirconate (in the absence of sintering aids). In an earlier work, it was proposed that barium loss during high temperature processing is responsible. ${ }^{7}$ Alternative explanations based on separation of the perovskite into two distinct phases have also been proposed. ${ }^{12}$ Here we probe the behavior of $20 \%$ yttrium doped barium zirconate compositions in which a precisely controlled level of barium deficiency is explicitly introduced. X-ray diffraction analysis permits construction of the ternary phase behavior in the $\mathrm{BaO}-\mathrm{ZrO}_{2}-\mathrm{YO}_{1.5}$ system in the vicinity of $\mathrm{BaZrO}_{3}$ at the sintering temperature of $1600^{\circ} \mathrm{C}$ and of a defect chemical picture of the accommodation of barium deficiency. The sharp, monotonic decrease in conductivity with increasing barium deficiency demonstrates that, irrespective of other contributions, cation non-stoichiometry plays a major role in the proton transport properties of yttrium-doped barium zirconate. 


\section{Experimental}

The Ba-deficient BYZ20 materials, $\mathrm{Ba}_{1-x} \mathrm{Zr}_{0.8} \mathrm{Y}_{0.2} \mathrm{O}_{3-\delta}$ (with target non-stoichiometries $x=0$ to 0.4 ), were synthesized via a chemical solution route described in detail elsewhere. ${ }^{8}$ The (nominal) compositions prepared are shown in the $\mathrm{BaO}-\mathrm{ZrO}_{2}$ $\mathrm{YO}_{1.5}$ ternary phase diagram, Fig. 1. Calcination of the powders obtained from the solution synthesis was carried out at $950{ }^{\circ} \mathrm{C}$ $(5 \mathrm{~h})$, a temperature at which substantial carbonate content remains and the crystallite size of the perovskite phase is $\sim 15 \mathrm{~nm}$. The multi-phase powder was compacted under isostatic pressure (300-370 MPa) and the resulting pellets sintered at $1600{ }^{\circ} \mathrm{C}$ for $24 \mathrm{~h}$ in flowing air. This reactive sintering procedure has been shown elsewhere to result in large-grained materials. ${ }^{8}$ To mitigate against $\mathrm{BaO}$ loss during the long exposure to high temperature, the pellets were additionally embedded in a powder of $\mathrm{BaCO}_{3}+$ Y-doped $\mathrm{BaZrO}_{3}$ during sintering. ${ }^{13}$

Compositions containing slight $\mathrm{Ba}$ excess were rather fragile after densification, preventing reliable conductivity measurements and limiting the analysis to $x \geq 0$. Based on similar behavior of Gd-doped barium cerate, ${ }^{14}$ it is likely that excess barium oxide residing in the grain boundary regions of the samples eventually reacts with atmospheric carbon dioxide and induces mechanical disintegration.

Phase analysis was performed directly on the sintered pellets (after light polishing) by X-ray diffraction using a Philips X'Pert Pro powder diffractometer $(\mathrm{CuK} \alpha, 45 \mathrm{kV}, 40 \mathrm{~mA})$. Rietveld refinement was carried out using the commercial software package Philips X'Pert Plus to determine lattice parameters and, for multi-phase compositions, phase fractions. The crosssectional microstructure of the as-fractured pellets was observed using LEO 1550VP field emission scanning electron microscopy (10 kV excitation voltage; In-lens mode; Carl Zeiss) after carbon coating. The average grain size of sintered pellets was determined using the mean linear intercept method. ${ }^{15}$ Quantitative elemental microanalyses of polished samples were conducted with a JEOL 8200 electron probe microanalyzer operated at $15 \mathrm{kV}$ and $25 \mathrm{nA}$ using a beam $10 \mu \mathrm{m}$ in diameter. The relative density of the pellets was measured by the Archimedes method using deionized water and in all cases was greater than $\sim 93 \%$ with respect to the theoretical density of undoped $\mathrm{BaZrO}_{3}\left(6.21 \mathrm{gcm}^{-3}\right)$.

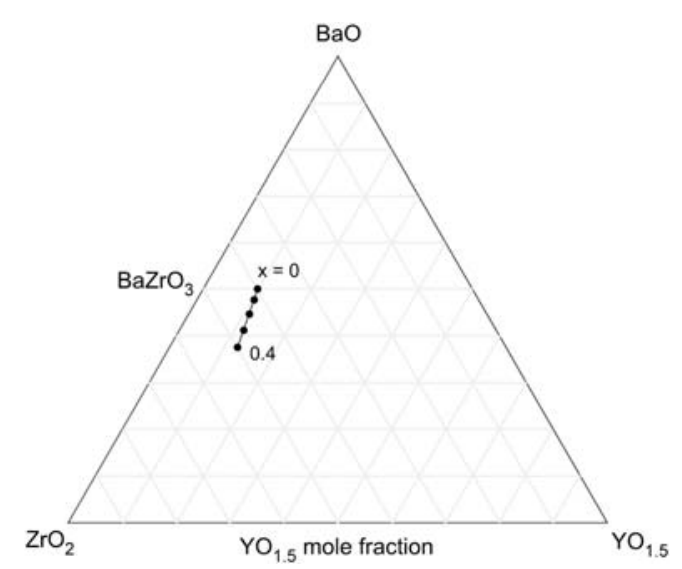

Fig. 1 Nominal composition of $\mathrm{Ba}_{1-x} \mathrm{Zr}_{0.8} \mathrm{Y}_{0.2} \mathrm{O}_{3-\delta}(x=0-0.4)$ pellets.
The conductivity of sintered pellets was measured by AC impedance spectroscopy under water saturated $\mathrm{N}_{2}$ or Ar atmosphere $\left(P_{\mathrm{H}_{2} \mathrm{O}}=0.031 \mathrm{~atm}\right)$ from 54 to $600{ }^{\circ} \mathrm{C}$ using an equilibration time of $30 \mathrm{~min}$ for each temperature. The measurement sequence was repeated several times until the resistivities at each temperature became equivalent from one measurement to the next. Data were collected in the frequency range from $10^{-1}$ to $1.3 \times 10^{7} \mathrm{~Hz}$ using a Solartron 1260 and a voltage amplitude of $20 \mathrm{mV}$. Silver electrodes were applied onto the surfaces of polished samples by brush painting colloidal silver paint (No. 16032, Ted Pella, Inc., Redding, CA).

The resulting impedance spectra were analyzed in terms of an equivalent circuit model in which (depending on the temperature regime) distinct RQ subcircuits were used to represent the grain interior and grain boundary regions. Here $\mathrm{R}$ is an ideal resistor with impedance $Z_{R}=R$, and $Q$ is a constant phase element with impedance $Z_{Q}=\left(Y(j \omega)^{n}\right)^{-1}$, where $j$ is $\sqrt{-1}$, $\omega$ is frequency, and $Y$ and $n$ are constants with $0<n<1$. Data analysis was performed using the software package Zview (Scribner Associates Inc., Southern Pines, NC).

\section{Results and discussions}

\subsection{Phase behavior}

The nominal and measured compositions of the sintered samples show a clear, linear correlation (Fig. 2), demonstrating that the Ba-deficiency, $x$, of pellets has been successfully controlled by the chemical synthesis. The measured sample composition is found to be slightly Ba-deficient relative to the nominal value by $\sim 0.02$ in all samples, despite the precautions taken to prevent $\mathrm{BaO}$ loss during sintering. ${ }^{8,13}$ The reasons for this small difference and whether it reflects a real difference between the nominal and actual compositions remain unclear, but the effect is slight enough that it is not considered further.

The X-ray diffraction patterns of sintered yttrium-doped barium zirconate pellets $\left(\mathrm{Ba}_{1-x} \mathrm{Zr}_{0.8} \mathrm{Y}_{0.2} \mathrm{O}_{3-\delta}\right)$ with Ba deficiency (Fig. 3) show that the primary phase for all compositions is the cubic perovskite. The data show no evidence of a tetragonal structure $^{6}$ or of phase separation. ${ }^{16,17} \mathrm{Up}$ to a deficiency of

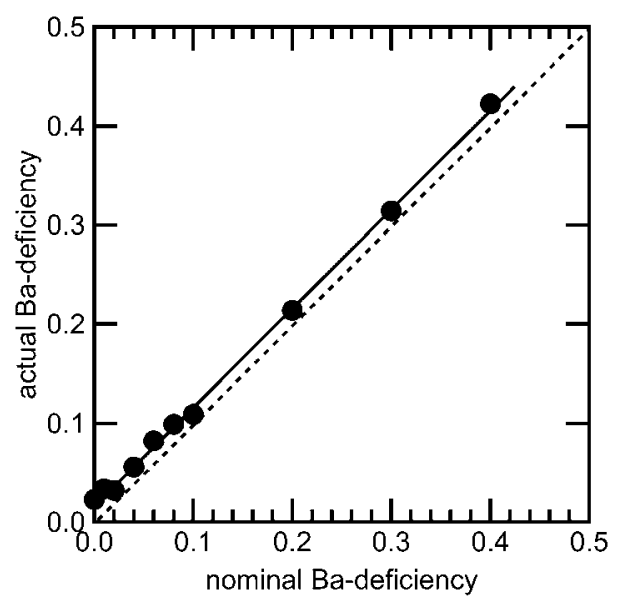

Fig. 2 Nominal and actual Ba-deficiency of yttrium-doped barium zirconate $\left(\mathrm{Ba}_{1-x} \mathrm{Zr}_{0.8} \mathrm{Y}_{0.2} \mathrm{O}_{3-\delta}\right)$ pellets sintered at $1600{ }^{\circ} \mathrm{C}$. 


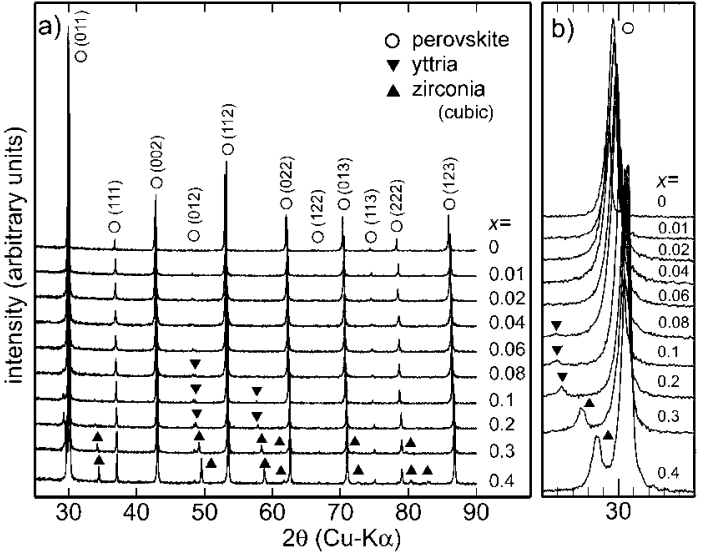

Fig. $3 \mathrm{X}$-Ray diffraction patterns of sintered yttrium-doped barium zirconate $\left(\mathrm{Ba}_{1-x} \mathrm{Zr}_{0.8} \mathrm{Y}_{0.2} \mathrm{O}_{3-\delta}\right)$ pellets with nominal $\mathrm{Ba}$ deficiency, $x$.

$x=0.06$, only the cubic perovskite is observed. In the composition region from $x=0.08$ to 0.2 zirconium-doped yttria (space group $I a \overline{3})^{18}$ emerges as a secondary phase, whereas in the region from 0.3 to 0.4 yttrium-doped zirconia (space group $F m \overline{3} m)^{19}$ emerges.

The phase and lattice constant behavior for these compositional regions are shown in Fig. 4. The lattice constant of the perovskite phase falls monotonically over the entire composition range examined, reaching a value comparable to that of undoped $\mathrm{BaZrO}_{3}(a=4.1973 \AA$ for ICSD database no. 90049) at $x \sim 0.2$. A similar correlation between perovskite cell constant and the presence of yttria has been reported for the case in which barium deficiency resulted as a consequence of processing conditions. ${ }^{13}$ The behavior observed here indicates the perovskite phase in the barium deficient region is largely yttrium-free, with most of the yttrium occurring in the secondary phases. In the two-phase

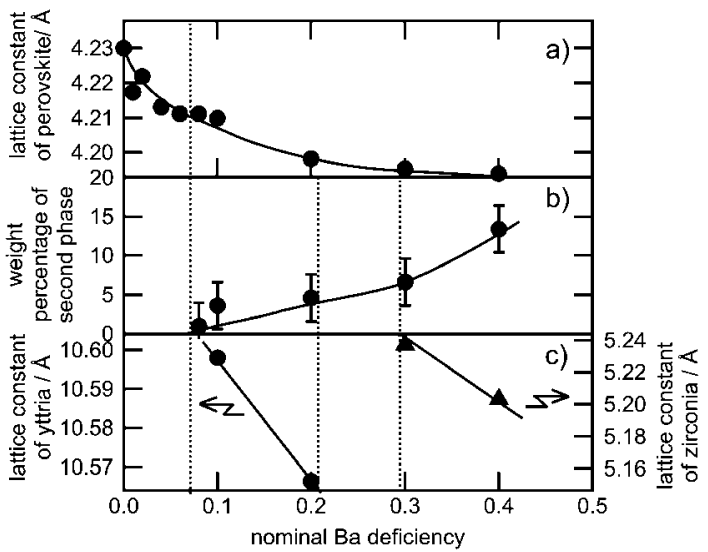

Fig. 4 Plots of (a) lattice constant of perovskite phase, (b) weight percentage of second phase and (c) lattice constants of yttria and zirconia cubic phases in sintered yttrium-doped barium zirconate pellets as a function of nominal Ba-deficiency. The minimum value of lattice constant for perovskite corresponds to undoped barium zirconate. The maximum and minimum lattice constants of yttria and zirconia in (c) correspond to the respective solubility limits. Dotted lines show phase boundaries. Uncertainty of data is drawn in error bar unless it is smaller than the size of symbol. region of perovskite plus yttria, the lattice constant of the yttria phase decreases, and similarly in the perovskite plus zirconia region, the lattice constant of the zirconia phase decreases. These lattice constant changes must reflect changes in composition, and most likely result from a change in the relative yttrium and zirconium concentrations in these phases rather than a change in barium content. The solubility of barium in yttria at $1600{ }^{\circ} \mathrm{C}$ lies below conventional detection limits, ${ }^{20}$ and, although the barium solubility in cubic zirconia has not been reported in the literature, its solubility in tetragonal zirconia is less than $\sim 0.5 \mathrm{~mol} \%$ at $1600{ }^{\circ} \mathrm{C}^{21}$ Thus, one can reasonably take the barium solubility in the non-perovskite phases of this system to be negligible. In such case, then, because the ionic radius of $\mathrm{Y}^{3+}$ is greater than that of $\mathrm{Zr}^{4+}\left(R_{\mathrm{Y} 3+}^{\mathrm{VI}}=0.9 \AA>R_{\mathrm{Zr} 4+}^{\mathrm{VI}}=0.72 \AA\right)$ the decreases in lattice constants correspond to a decreasing concentration of $\mathrm{Y}^{3+}$ with increasing $x$, first in the yttria phase and then in the zirconia phase. The change in the nature of the secondary phase, from yttria to zirconia, is consistent with a decrease in yttrium content and hence an increase in zirconium content in the non-perovskite phases. That is, as $x$ increases beyond 0.2 and the $\mathrm{Zr}$ content in the yttria phase increases, eventually reaching the solubility limit, and zirconia becomes the equilibrium secondary phase.

Based on these observations and, again, the assumption that the $\mathrm{Ba}$ solubility in the doped yttria and zirconia phases is negligible, the pseudo-ternary phase behavior of the $\mathrm{BaO}-\mathrm{ZrO}_{2}$ $\mathrm{YO}_{1.5}$ system can be estimated (Fig. 5). The well-documented linear relationship between lattice constant and composition within the $\mathrm{Zr}$-doped yttria and Y-doped zirconia systems ${ }^{22}$ enables determination of the composition of these phases in the two-phase regions whenever they occur. The lattice constant of yttria in the $x=0.2$ sample (Fig. 4c) implies a composition that is close to the maximum $\mathrm{Zr}$ solubility at $1600{ }^{\circ} \mathrm{C},{ }^{22}$ suggesting that the phase diagram represents equilibrium at this temperature. Overall, the phase diagram generated here is quite consistent with that reported at $1600{ }^{\circ} \mathrm{C}$ in the $\mathrm{BaO}-\mathrm{ZrO}_{2}-\mathrm{ScO}_{1.5}$ system and also that very recently suggested for the $\mathrm{BaO}-\mathrm{ZrO}_{2}-\mathrm{YO}_{1.5}$ system, in which the solubility of $\mathrm{Ba}$ in $\mathrm{Y}$-stabilized cubic zirconia was found (in both cases) to lie below the detection limit. ${ }^{2324}$ Given

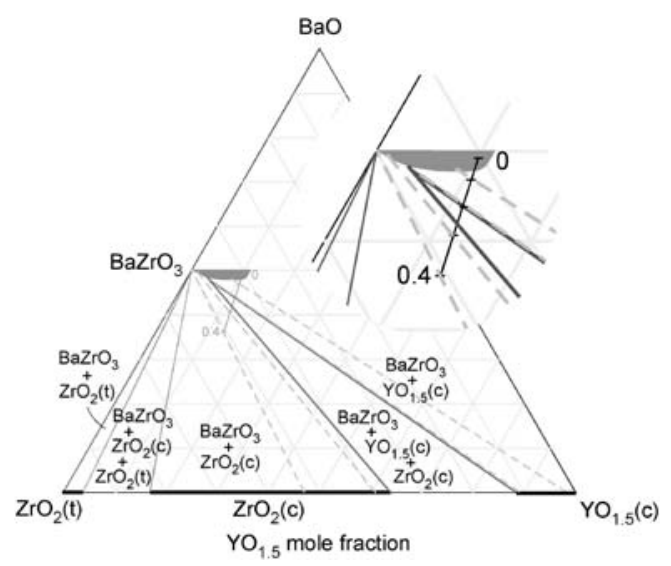

Fig. 5 Estimated pseudo-ternary phase diagram in $\mathrm{BaO}-\mathrm{ZrO}_{2}-\mathrm{YO}_{1.5}$ system, with area around $\mathrm{BaZrO}_{3}$ expanded in inset. Tie lines are drawn as dotted lines. Single phase barium zirconate along the composition $\left(\mathrm{Ba}_{1-x} \mathrm{Zr}_{0.8} \mathrm{Y}_{0.2} \mathrm{O}_{3-\delta}\right)$ occurs up to $x=0.06$, drawn as grey area. The remaining boundaries for the single phase region are uncertain. 
the chemical similarity of yttrium and scandium - both are group 3 elements, they possess the same electron configuration in their valence shell, and they show almost complete solid solution in both metal ${ }^{25}$ and oxide ${ }^{26}$ forms - such a similarity in the ternary phase behaviors is to be expected.

The pseudo-ternary phase diagram reveals that the matrix perovskite composition follows a non-linear path as $x$ increases in the overall system composition. Between $x=0$ and 0.06 , the perovskite phase occurs as a single phase, and its composition is (by definition) simply that of the overall composition. At larger values of $x,(0.08 \leq x \leq 0.4)$, the system enters a two-phase region and the composition of the perovskite phase tracks the phase boundary with the secondary phase, deviating towards low yttrium content relative to the average composition. Such behavior is consistent with the observation that the highly Ba-deficient compositions yield a perovskite phase with a lattice constant essentially identical to that of undoped barium zirconate.

It is of some value to consider the means by which the perovskite structure accommodates barium deficiency in the singlephase region. Undoped barium zirconate tolerates negligible barium deficiency, as captured in the phase diagram, Fig. 5. This behavior indicates that A-site vacancies are unfavorable in $\mathrm{BaZrO}_{3}$, as are other alternative mechanisms of accommodating barium deficiency. The contrasting accommodation of barium deficiency in the doped system indicates that the dopant itself must play a major role in stabilizing the non-stoichiometric structure. These observations suggest that, as in doped barium cerate,${ }^{14}$ non-stoichiometry in yttrium doped barium zirconate involves dopant partitioning over both the $\mathrm{A}$ and $\mathrm{B}$ sites of the perovskite phase. Taking the limit of the single phase region to occur at $x=0.07$ and taking this stoichiometry to correspond to fully occupied cation sites, the perovskite formula becomes $\left(\mathrm{Ba}_{0.964} \mathrm{Y}_{0.036}\right)\left(\mathrm{Zr}_{0.829} \mathrm{Y}_{0.171}\right) \mathrm{O}_{3-\delta}$, with $17.5 \%$ of the $\mathrm{Y}$ incorporated on the A-site and a $\delta$ of only 0.675 , just over half of what it would be for a comparable level of doping and $x=0$. An implication of this interpretation is that the decrease in perovskite cell constant with increasing $x$ occurs as a result of replacing a portion of the relatively large barium cations $\left[R_{\mathrm{Ba} 2+}^{\mathrm{XII}}=1.61 \AA\right]$ with smaller yttrium cations $\left[R_{\mathrm{Y} 3+}^{\mathrm{IX}}=1.08 \AA\right]^{27}$ while simultaneously increasing the concentration of small zirconium cations on the B-site. The alternative possibilities of A-site vacancy creation or (especially) cation interstitial site occupancy (by $\mathrm{Y}$ or $\mathrm{Zr}$ ) would not be expected to produce the observed lattice constant change. In terms of the anionic defects relevant to water uptake and proton conductivity, yttrium incorporation onto the A-site directly lowers the concentration of oxygen vacancies and thereby presumably also the proton concentration upon hydration. A somewhat surprising feature of yttria-doped barium zirconate is that the barium deficiency leads to yttria rather than zirconia exsolution, ${ }^{28}$ which implies that the perovskite very quickly (i.e. at small $x$ ) becomes undoped and hence displays a negligible anion defect concentration. These connections between barium deficiency and anion vacancy concentration thus provide a link between cation stoichiometry and proton conductivity.

\subsection{Microstructural characteristics}

The microstructure and average grain sizes of the Ba-deficient BYZ20 samples are shown in Fig. 6 and 7, respectively. The relatively large grain size in the stoichiometric material $(\sim 1 \mu \mathrm{m})$ is, as presented elsewhere, ${ }^{8}$ a result of the atypical processing methodology in which the green body is obtained from powders that are not fully calcined. Following a more conventional approach, similar sintering conditions to those employed here typically yield grains with an average size of only $\sim 0.5 \mu \mathrm{m} .^{7,8,13}$ More relevant to the present study is the clear decrease in grain size with increasing barium deficiency, which rapidly falls to $\sim 0.5 \mu \mathrm{m}$ at $x=0.02$. The decrease in grain size suggests lower cation diffusion rates ${ }^{29}$ with increasing $x$, which, in turn, suggests a decrease in point defect concentrations, particular cationic point defects, in the barium deficient samples. The result is consistent with the proposal that barium deficiency is accommodated not by A-site vacancies (or $\mathrm{Y}^{3+}$ or $\mathrm{Zr}^{4+}$ interstitials), which would lead to an increase in cation diffusivity, but rather by dopant partitioning over the A and B sites. The decrease in anion vacancy concentration (relative to the stoichiometric composition) implied by such partitioning could plausibly decrease cation mobility. ${ }^{30}$

\subsection{Bulk conductivity}

Impedance spectra for selected compositions are shown in Fig. 8 in Nyquist form. In general, the spectra displayed multiple arcs, as

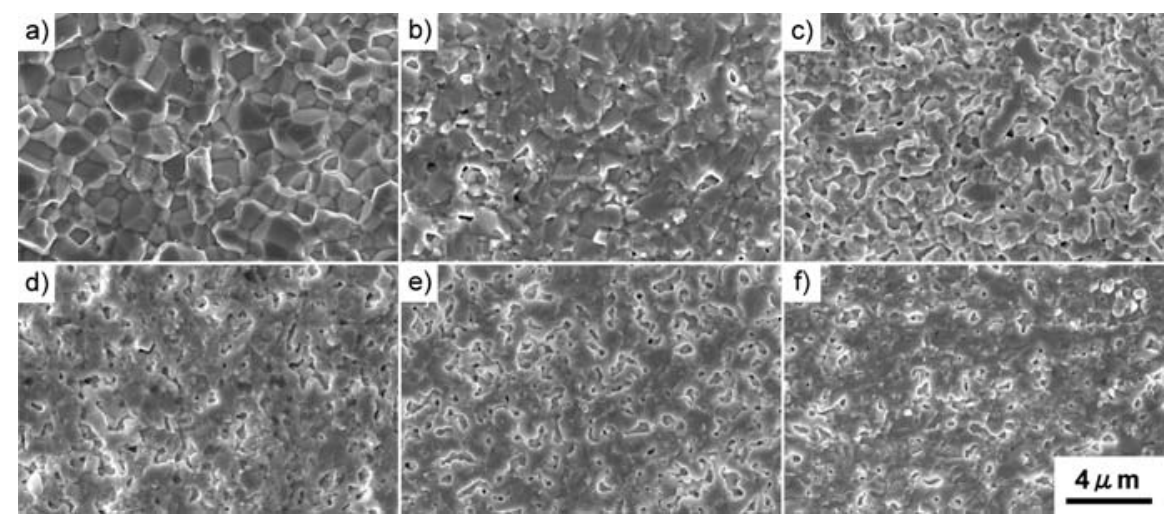

Fig. 6 Cross-sectional scanning electron microscopy images of sintered yttrium-doped barium zirconates $\left(\mathrm{Ba}_{1-x} \mathrm{Zr}_{0.8} \mathrm{Y}_{0.2} \mathrm{O}_{3-\delta}\right)$ : (a) $x=0$, (b) $x=0.01$, (c) $x=0.02$, (d) $x=0.04$, (e) $x=0.06$ and (f) $x=0.1$. 


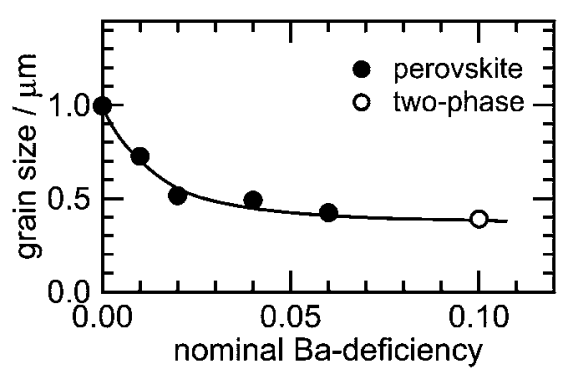

Fig. 7 Plot of grain size of sintered yttrium-doped barium zirconates $\left(\mathrm{Ba}_{1-x} \mathrm{Zr}_{0.8} \mathrm{Y}_{0.2} \mathrm{O}_{3-\delta}\right)$ as a function of nominal Ba-deficiency, $x$.

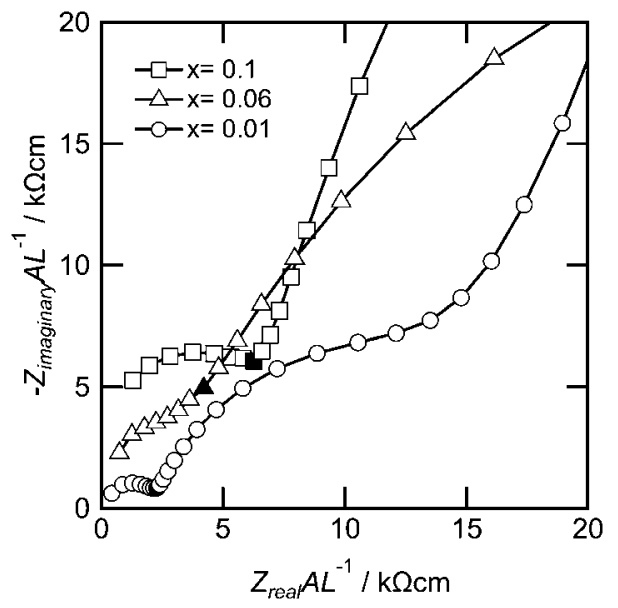

Fig. 8 Impedance spectra collected at $200{ }^{\circ} \mathrm{C}$ from Ba-deficient samples of $x=0.01,0.06$ and 0.1 . Closed symbols represent experimental data collected at frequencies of $901 \mathrm{kHz}(x=0.01), 732 \mathrm{kHz}(x=0.06)$, and $896 \mathrm{kHz}(x=0.1)$. For visibility, impedance spectra for $x=0.06$ and 0.1 are offset along y axis by 1.5 and $3 \mathrm{k} \Omega \mathrm{cm}$, respectively.

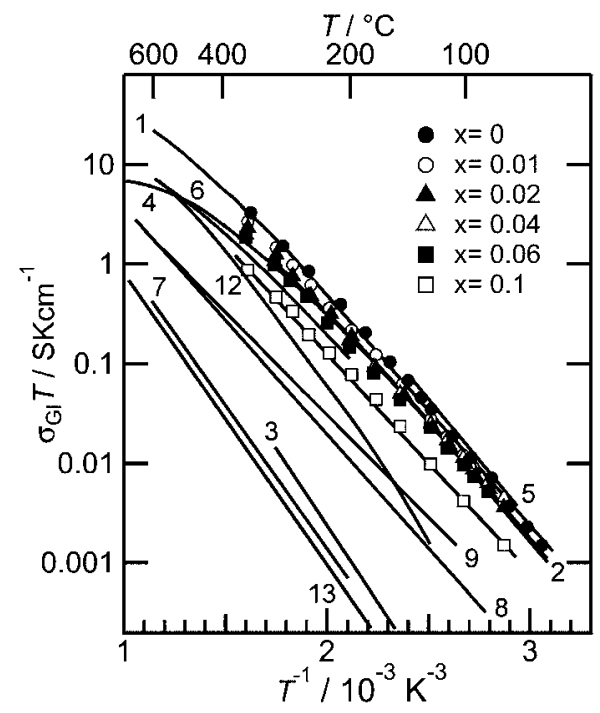

Fig. 9 Arrhenius plots of bulk conductivities in yttrium-doped barium zirconates as a function of Ba-deficiency, $x$. Legend information is provided in Table 2. is typical for this class of proton conductors when measured at moderate temperatures. The highest frequency arc corresponds to bulk (grain interior) transport, the intermediate frequency arc to grain boundary transport, and the lowest frequency response(s) to electrochemical processes at the interface. ${ }^{31}$ The bulk conductivities implied from the fitting to the spectra for each of the $\mathrm{Ba}_{1-x} \mathrm{Zr}_{0.8} \mathrm{Y}_{0.2} \mathrm{O}_{3-\delta}$ compositions within the single phase region are presented in Arrhenius form in Fig. 9, along with one representative example from the two-phase region $(x=0.1)$. Selected literature results are also presented for comparison. ${ }^{5,6,8,12,13,32-34}$

It is evident from the data in Fig. 9 that the conductivity decreases with increasing Ba deficiency, in line with the interpretation presented here of the phase behavior. The role of stoichiometry on transport properties is more clearly visible in the conductivity isotherm for a temperature of $200{ }^{\circ} \mathrm{C}$ (the plotted values are obtained by interpolation from measurements close to this temperature), Fig. 10 . In the single-phase region $(0 \leq$ $x \leq 0.06$ ), the bulk conductivity gradually and monotonically decreases with increasing Ba deficiency, falling by a factor of 2 across this composition range. The conductivity of the two-phase sample is even lower, and the drop between $x=0.06$ and $x=0.1$ is about another factor of two. The data in Table 1, in which the transport properties are summarized, furthermore reveal that the activation enthalpy for bulk migration $\left(\Delta H_{\mathrm{GI}}\right)$ decreases slightly with increasing $x$, as does the pre-exponential factor $\left(A_{\mathrm{GI}}\right)$, where these quantities are defined according to

$$
\sigma T=A \exp \left(\frac{-\Delta H}{k_{b} T}\right)
$$

where $\sigma$ is conductivity, $T$ is temperature, and $k_{\mathrm{b}}$ is Boltzmann's constant. The pre-exponential term, evaluated over the temperature region $\sim 80$ to $270{ }^{\circ} \mathrm{C}$, reflects a number of transport terms (hydration entropy, migration entropy, jump distance, etc.) of which the concentration of anion vacancies in the dry state available for water incorporation might be expected to vary most significantly with $x$. That is, a decrease in $A_{\mathrm{GI}}$ can very plausibly result from a decrease in bulk proton content, in turn, resulting from the dopant partitioning over the two cation sites. However, it is not possible to meaningfully predict the dependence of proton concentration on $x$ (even at a given temperature and given hydration thermodynamic parameters) because the relative concentration of A-site vacancies and A-site incorporated

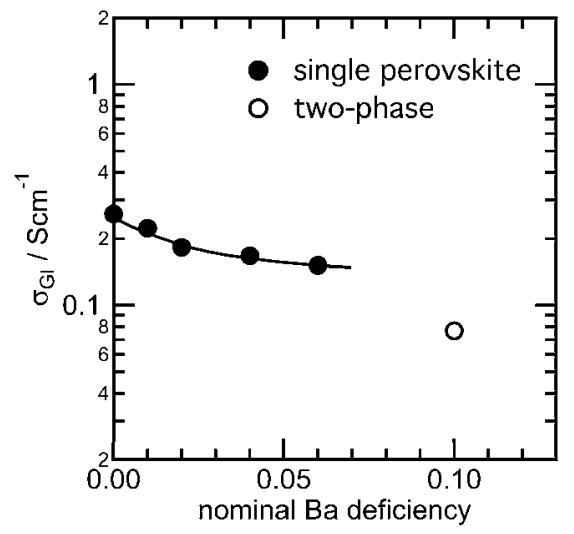

Fig. 10 Bulk conductivities calculated at $200^{\circ} \mathrm{C}$ from Arrhenius plots in Ba-deficient BYZ20. 
Table 1 Chemical composition, grain size and proton-conduction parameters of Ba-deficient yttrium-doped barium zirconates $\left(\mathrm{Ba}_{1-x} \mathrm{Zr}_{0.8} \mathrm{Y}_{0.2} \mathrm{O}_{3-\delta}\right)$

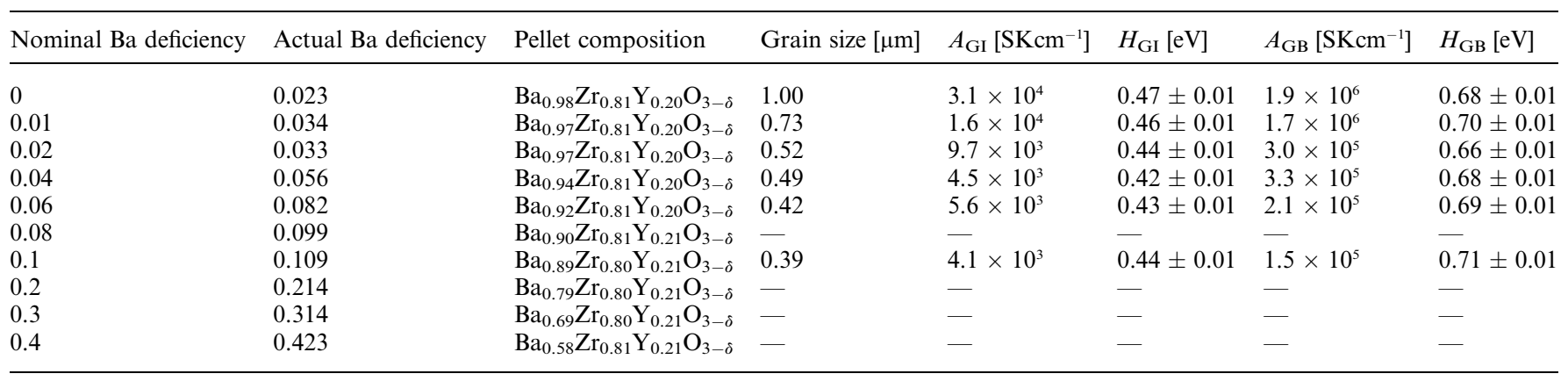

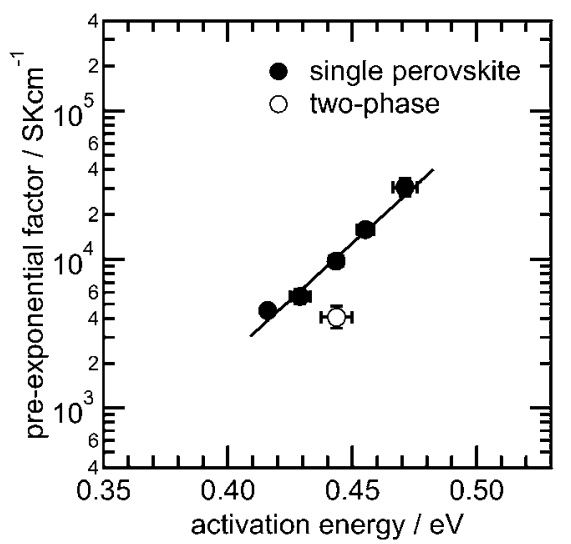

Fig. 11 Plot of pre-exponential factor and activation energy for bulk conductivities in Ba-deficient BYZ20.

yttrium species is not known. The impact of cation non-stoichiometry on other terms relating to proton motion, whether to increase or decrease $A_{\mathrm{GI}}$, is not immediately obvious.

Turning away from a defect chemical analysis, a simultaneous examination of the behavior of $A_{\mathrm{GI}}$ and $\Delta H_{\mathrm{GI}}$ reveals that, for samples within the single-phase composition region, $x \leq 0.06$, there is a clear linear correlation between $\log \left(A_{\mathrm{GI}}\right)$ and $\Delta H_{\mathrm{GI}}$, plotted as parametric functions of composition, Fig. 11. This behavior is reminiscent of the Zener empirical rule for the correlation between entropy and enthalpy ${ }^{35}$ of diffusion in solids. The data for the two-phase material is clearly displaced from this line, consistent with an overall change in phase behavior. While the fundamental reason for the correlation is not apparent, the result emphasizes the difference between the single-phase and two-phase compositions.

Returning to the overall dependence of conductivity on composition, Fig. 10, the relatively sharp drop in conductivity as $x$ is increased to 0.10 is consistent with the occurrence, as indicated on the phase diagram (Fig. 7), of a perovskite phase that is almost entirely undoped and a yttria phase with negligible conductivity at $200{ }^{\circ} \mathrm{C}$. However, the change in conductivity on traversing the two-phase region is not as extreme as that reported earlier by Babilo et al. ${ }^{13}$ in which a decrease by two orders of magnitude was observed when samples were exposed to sintering conditions that facilitated $\mathrm{BaO}$ evaporation. Indeed, although the literature, summarized in Table 2, hints towards a decrease in conductivity with increasingly harsh sintering conditions (which would favor $\mathrm{BaO}$ loss), there is no clear correlation between processing conditions and transport properties. Accordingly, other factors beyond A:B cation stoichiometry, for example, enhanced impurity incorporation under extreme conditions, or possible structural variations that appear under fabrication conditions differing from those employed here, must play a role in establishing the conductivity.

\subsection{Grain boundary and total conductivity}

Analogous to the bulk conductivity, barium deficiency has a detrimental impact on both the total (or effective) grain boundary conductivity, $\sigma_{\mathrm{GB}}$, and the total conductivity, $\sigma_{\text {total }}$, as evident in Fig. 12 and 13, respectively. At low temperatures,

Table 2 Characteristics of yttrium-doped barium zirconates shown in Figs. 9, 12 and 13

\begin{tabular}{|c|c|c|c|c|c|c|c|c|}
\hline No. & $\begin{array}{l}\text { Y content } \\
\text { [at } \% \text { ] }\end{array}$ & $\begin{array}{l}\text { Sintering } \\
\text { temperature }\left[{ }^{\circ} \mathrm{C}\right]\end{array}$ & $\begin{array}{l}\text { Sintering } \\
\text { time }[\mathrm{h}]\end{array}$ & $\begin{array}{l}\text { Actual composition } \\
\text { of sintered pellet }\end{array}$ & $\begin{array}{l}\mathrm{Ba} \\
\text { deficiency }\end{array}$ & $\begin{array}{l}\text { Grain } \\
\text { size }[\mu \mathrm{m}]\end{array}$ & Authors & Reference \\
\hline 1 & 20 & 1600 & 24 & $\mathrm{Ba}_{0.98} \mathrm{Zr}_{0.81} \mathrm{Y}_{0.20} \mathrm{O}_{3-\delta}$ & 0.02 & 1.00 & Yamazaki et al. & 8 \\
\hline 2 & 20 & 1600 & 24 & $\mathrm{Ba}_{1.00} \mathrm{Zr}_{0.81} \mathrm{Y}_{0.19} \mathrm{O}_{3-\delta}$ & - & 0.46 & Babilo et al. & 13 \\
\hline 3 & 20 & 1600 & 16 & $\mathrm{Ba}_{0.97} \mathrm{Zr}_{0.82} \mathrm{Y}_{0.19} \mathrm{O}_{3-\delta}$ & 0.05 & - & Babilo et al. & 13 \\
\hline 4 & 10 & 1715 & 30 & - & - & - & Bohn and Schober & 5 \\
\hline 5 & 20 & 1700 & - & - & - & - & Kreuer et al. & 6 \\
\hline 6 & 5 & 1800 & 20 & $\mathrm{Ba}_{0.99} \mathrm{Zr}_{0.95} \mathrm{Y}_{0.05} \mathrm{O}_{3-\delta}$ & 0.01 & 0.67 & Iguchi et al. & 32,33 \\
\hline 7 & 5 & 1800 & 200 & $\mathrm{Ba}_{0.97} \mathrm{Zr}_{0.97} \mathrm{Y}_{0.05} \mathrm{O}_{3-\delta}$ & 0.05 & 1.61 & Iguchi et al. & 32,33 \\
\hline 8 & 10 & $\sim 2200$ & - & - & - & - & Duval et al. & 34 \\
\hline 9 & 10 & 1720 & 24 & - & - & - & Duval et al. & 34 \\
\hline 10 & 20 & 1500 & 20 & - & - & $\sim 0.2$ & Cervera et al. & 37 \\
\hline 11 & 20 & 1250 & 10 & - & - & $\sim 0.05$ & Cervera et al. & 37 \\
\hline 12 & 10 & 1500 & - & - & - & - & Azad et al. & 12 \\
\hline 13 & 10 & $1500+1720$ & - & - & - & - & Azad et al. & 12 \\
\hline
\end{tabular}




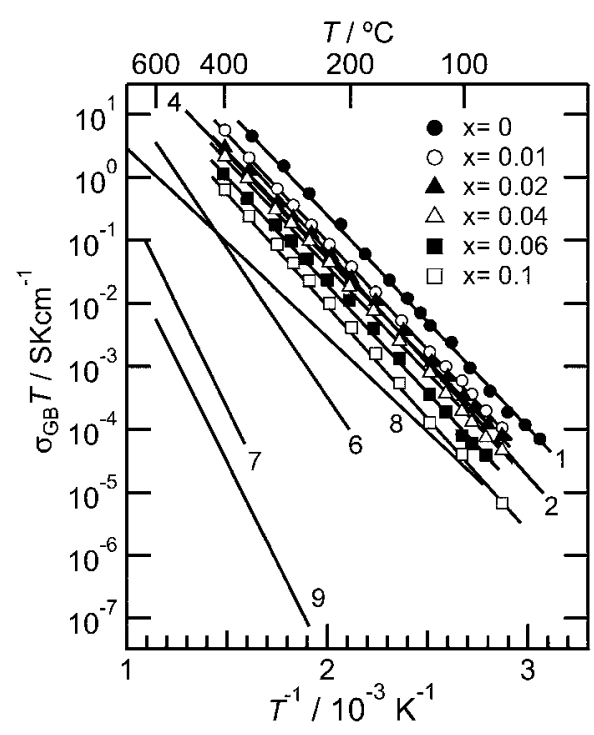

Fig. 12 Temperature dependence of grain boundary conductivity in Badeficient BYZ20. Legend information is provided in Table 2.

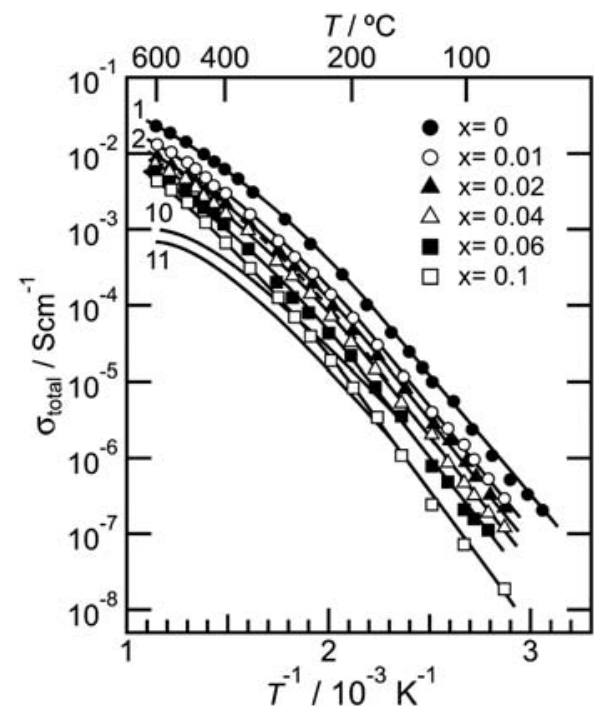

Fig. 13 Temperature dependence of total conductivity in Ba-deficient BYZ20. Legend information is provided in Table 2.

transport across the material is dominated by the highly resistive grain boundaries. At $x=0.1$ both the grain boundary and total conductivities display a factor of $\sim 30$ decrease in magnitude (at $200{ }^{\circ} \mathrm{C}$ ) relative to the stoichiometric composition, much greater than the factor of 4 decrease noted for bulk transport. At high temperatures, because the activation energy for grain boundary transport is higher than that for bulk transport, the bulk becomes rate-limiting and the total conductivity is dominated by the grain interior properties. In this regime, specifically at $600^{\circ} \mathrm{C}$, the total conductivity decreases by a factor of $\sim 5$ from $x=0$ to $x=0.1$.

In a polycrystalline sample, the total or effective grain boundary conductance extracted directly from the impedance analysis is given by the inverse of the resistance across an individual grain boundary multiplied by the number of grain boundaries traversed, $n^{8,36}$ Because the microstructure varies

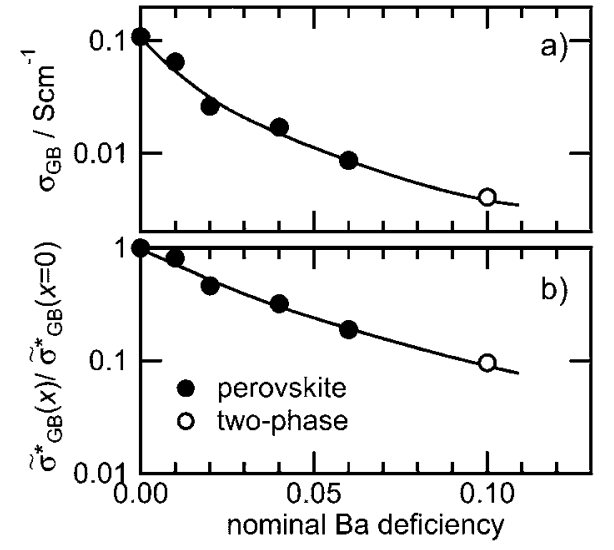

Fig. 14 Impact of Ba-deficiency on grain boundary conductivities in yttrium-doped barium zirconates. (a) Grain boundary conductivities calculated at $200{ }^{\circ} \mathrm{C}$ from Arrhenius plots and (b) proton conductivities per grain boundary at nominal Ba-deficiency, $x$, relative to stoichiometry.

across the samples represented in Fig. 12, with grain size monotonically decreasing with $x$, it is of value to eliminate the microstructural influence and compare the per boundary properties to assess the influence of composition on inherent transport behavior. As $n$ is inversely proportional to the mean grain size, $D$, the specific (or inherent) grain boundary conductivity, $\sigma_{G B}^{*}$, is proportional to $\sigma_{G B} / D$, where the proportionality factor is the (unknown) grain boundary thickness, $d$. While the latter can be estimated from an examination of the dielectric behavior, the procedure relies on assumptions about grain boundary behavior, and such a detailed analysis is not necessary for the present purpose of determining the influence of composition on the grain boundary properties. Instead, we define here the area specific grain boundary conductance,

$$
\tilde{\sigma}_{G B}^{*}=\frac{\sigma_{G B}^{*}}{d}=\frac{\sigma_{G B}}{D}
$$

and evaluate the relative change in this quantity as a function of composition, as well as that of the directly accessible quantity, $\sigma_{G B}$, Fig. 14.

The data in Fig. 14 immediately reveal that not only does the total grain boundary conductivity decrease with increasing barium deficiently, but also does the specific grain boundary conductivity. Thus, both microstructure and inherent transport properties contribute to the increased grain boundary resistance of barium deficient samples, with the inherent compositional effect being the more important contributor (assuming a fixed grain boundary thickness). This observation indicates that ensuring barium zirconate materials with high overall conductivity, particularly at moderate temperatures, will require both rigorous stoichiometry control and enhanced grain growth.

\section{Conclusions}

Cation non-stoichiometry (i.e., barium deficiency) of yttriumdoped barium zirconate materials has been accurately controlled using a chemical solution synthesis method. Materials of nominal composition $\mathrm{Ba}_{1-x} \mathrm{Zr}_{0.8} \mathrm{Y}_{0.2} \mathrm{O}_{3-\delta}$ were examined over the range $x=0$ to 0.4 . A single perovskite phase appears up to 
$x=0.06$, followed by two types of two-phase regions with increasing $x$. In the range $0.08 \leq x \leq 0.2$ yttria appears as a secondary phase along with the primary perovskite, whereas in the range $0.3 \leq x \leq 0.4$ zirconia is the secondary phase. With increasing $\mathrm{Ba}$ deficiency the lattice constant of the majority perovskite phase monotonically decreases, attaining a value at $x=0.3$ and 0.4 comparable to that of undoped barium zirconate. Thus, the excess B-site cations at large values of $x$ are accommodated in the system by exsolution of yttria. The estimated pseudo-ternary phase diagram in the $\mathrm{BaO}-\mathrm{ZrO}_{2}-\mathrm{YO}_{1.5}$ system shows that there are two paths that the matrix perovskite composition follows in response to increasing barium deficiency; (1) tracking the overall composition in the single-phase region $(0 \leq x \leq 0.06)$ and (2) straying from the overall composition and tracking instead the phase boundary between the perovskite and secondary phases towards undoped barium zirconate $(0.08 \leq x$ $\leq 0.4$ ). The mean grain size of sintered compacts is also sensitive to the Ba deficiency. The grain size decreases from $\sim 1 \mu \mathrm{m}$ for the nominally stoichiometric material to $\sim 0.5 \mu \mathrm{m}$ at just $0.02 \mathrm{Ba}$ deficiency.

The most significant outcome of this work is the observation of a clear detrimental impact from barium deficiency on the proton conductivity of yttrium-doped barium zirconate. In the single phase region, the grain interior proton conductivity monotonically decreases with an increase in Ba deficiency, falling by a factor of $\sim 2$ from $x=0$ to $x=0.06$. It is proposed that dopant partitioning over both $\mathrm{A}$ and $\mathrm{B}$ sites accounts for the decrease in both the bulk conductivity and the lattice constant of the perovskite phase. On traversing the boundary into the two-phase region of perovskite and yttria, the drop in conductivity is sharp, exceeding the decrease that would be expected from an extrapolation of the behavior in the single-phase region. Specifically, in the two-phase material with $x=0.1$ (having $\sim 3$ mass $\%$ yttria) the grain interior conductivity is approximately half that of the closest single-phase composition $(x=0.06)$. Furthermore, the Arrhenius parameters obtained in the two-phase region do not obey the Zener empirical rule observed in the single perovskite region. The abrupt change of bulk transport behavior in the twophase region coincides with the alteration of the path of the perovskite composition in the ternary phase diagram as $x$ is increased, from one which coincides with the average composition to one that tends towards undoped barium zirconate.

The detrimental impact of Ba deficiency on the grain boundary transport is found to be substantially larger than on bulk transport. For example, the grain boundary conductivity decreases by a factor of $\sim 30$, opposed to $\sim 4$ at $x=0.1$ and $200{ }^{\circ} \mathrm{C}$ (relative to $x=0$ ). This large effect reflects both an increase in the number density of grain boundaries, by a factor of about 3 at $x=0.1$ relative to $x=0$, and an increase in the resistance across individual grain boundaries, by about one order of magnitude. Thus, although the inherent properties of the grain boundaries vary more sensitively with $x$ than does grain size, the impact on the latter is not insignificant.

The combined contributions of bulk and grain boundary conductivity lead to total conductivities that curve downward at high temperature in an Arrhenius representation. This is readily understood to reflect transport that is dominated by grain boundaries at low temperatures, with high activation energy, and controlled by both grain boundaries and grain interiors at high temperatures, with reduced activation energy. At all temperatures, increasing barium deficiency leads to decreasing conductivity. These results demonstrate that precise stoichiometry control in addition to enhancing grain growth is required to attain yttrium doped barium zirconate materials that display attractive transport properties. The reactive sintering approach, even in the absence of sintering aids, is an effective means for achieving both objectives and reproducibly results in large-grained, stoichiometric $\mathrm{BaZr}_{0.8} \mathrm{Y}_{0.2} \mathrm{O}_{3-\delta}$ with a total conductivity of $1 \times 10^{-2} \mathrm{Scm}^{-1}$ at $450{ }^{\circ} \mathrm{C}$.

\section{Acknowledgements}

This work was supported by the Gordon and Betty Moore Foundation and by JSPS Postdoctoral Fellowships for Research Abroad. Additional support was provided by the National Science Foundation through the Caltech Center for the Science and Engineering of Materials, a Materials Research Science and Engineering Center (DMR-052056). The authors thank Dr Chi $\mathrm{Ma}$ for assistance with scanning electron microscopy and electron probe microanalysis.

\section{References}

1 H. Iwahara, Solid State Ionics, 1996, 86-88, 9-15.

2 T. Norby, Solid State Ionics, 1999, 125, 1-11.

3 K. D. Kreuer, Annu. Rev. Mater. Res., 2003, 33, 333-359.

4 S. M. Haile, Acta Mater., 2003, 51, 5981-6000.

5 H. G. Bohn and T. Schober, J. Am. Ceram. Soc., 2000, 83, 768-772.

6 K. D. Kreuer, S. Adams, W. Munch, A. Fuchs, U. Klock and J. Maier, Solid State Ionics, 2001, 145, 295-306.

7 S. Imashuku, T. Uda and Y. Awakura, Electrochem. Solid-State Lett., 2007, 10, B175-B178.

8 Y. Yamazaki, R. Hernandez-Sanchez and S. M. Haile, Chem. Mater., 2009, 21, 2755-2762.

9 P. Babilo and S. M. Haile, J. Am. Ceram. Soc., 2005, 88, 2362-2368.

10 S. W. Tao and J. T. S. Irvine, Adv. Mater., 2006, 18, 1581-1584.

11 J. H. Tong, D. Clark, M. Hoban and R. O'Hayre, Solid State Ionics, 2010, 181, 496-503.

12 A. K. Azad, C. Savaniu, S. W. Tao, S. Duval, P. Holtappels, R. M. Ibberson and J. T. S. Irvine, J. Mater. Chem., 2008, 18, 3414-3418.

13 P. Babilo, T. Uda and S. M. Haile, J. Mater. Res., 2007, 22, 13221330.

14 D. Shima and S. M. Haile, Solid State Ionics, 1997, 97, 443-455.

15 A. M. Gokhale, in ASM handbook, ed. G. F. VanderVoort, ASM International, Ohio, 2004, pp. 428-443.

16 A. Kojima, K. Tanaka, Y. Oyama, T. Higuchi and S. Yamaguchi, in The 31st Symposium on Solid State Ionics, The Mining and Materials Processing Institute of Japan, Niigata, Japan, 2005, p. 157.

17 S. Imashuku, T. Uda, Y. Nose, K. Kishida, S. Harada, H. Inui and Y. Awakura, J. Electrochem. Soc., 2008, 155, B581-B586.

18 G. Baldinozzi, J. F. Berar and G. Calvarin, Mater. Sci. Forum, 1998, 278-281, 680-685.

19 H. Horiuchi, A. J. Schultz, P. C. Leung and J. M. Williams, Acta Crystallographica B, 1984, 40, 367-372.

20 K. N. Marushkin, G. D. Nipan and V. B. Lazarev, Inorganic Materials, 1994, 30, 475-479.

21 J. O. A. Paschoal, H. Kleykamp and F. Thummler, J. Nucl. Mater., 1987, 151, 10-21.

22 V. S. Stubican, R. C. Hink and S. P. Ray, J. Am. Ceram. Soc., 1978, 61, 17-21.

23 S. Imashuku, T. Uda, Y. Nose and Y. Awakura, Journal of Phase Equilibria and Diffusion, 2010, 31, 348-356.

24 S. Imashuku, T. Uda, T. Ichitsubo, E. Matsubara and Y. Awakura, J. Phase Equilib. Diffus., 2007, 28, 517-522.

25 B. J. Beaudry and A. H. Daane, Trans. Metall. Soc. AIME, 1963, 227, 865-868.

26 W. Trzebiat and R. Horyn, B Acad Pol Sci-Chim, 1965, 13, 311. 
27 R. D. Shannon, Acta Crystallogr., Sect. A: Cryst. Phys., Diffr., Theor. Gen. Crystallogr., 1976, 32, 751-767.

28 A. Magrez and T. Schober, Solid State Ionics, 2004, 175, 585-588.

29 S.-J. L. Kang, Sintering: densification, grain growth, and microstructure, Elsevier, Oxford, 2005.

30 J. L. Routbort, S. J. Rothman, N. Chen, J. N. Mundy and J. E. Baker, Phys. Rev. B: Condens. Matter, 1991, 43, 5489-5497.

31 E. Barsoukov and J. R. Macdonald, Impedance Spectroscopy: Theory, Experiment, and Applications, John Wiley\& Sons, Inc., Honoken, 2005.

32 F. Iguchi, T. Yamada, N. Sata, T. Tsurui and H. Yugami, Solid State Ionics, 2006, 177, 2381-2384.
33 F. Iguchi, T. Tsurui, N. Sata, Y. Nagao and H. Yugami, Solid State Ionics, 2009, 180, 563-568.

34 S. B. C. Duval, P. Holtappels, U. F. Vogt, E. Pomjakushina, K. Conder, U. Stimming and T. Graule, Solid State Ionics, 2007, 178, $1437-1441$

35 W. Shockley, J. H. Hollomon, R. Maurer and F. Seitz, Imperfections in Nearly Perfect Crystals, John Wiley \& Sons, Inc., New York, 1952.

36 S. M. Haile, D. L. West and J. Campbell, J. Mater. Res., 1998, 13, 1576-1595.

37 R. B. Cervera, Y. Oyama, S. Miyoshi, K. Kobayashi, T. Yagi and S. Yamaguchi, Solid State Ionics, 2008, 179, 236-242. 